\title{
ИСПОЛЬЗОВАНИЕ ПРЕИМУЩЕСТВ НИЗКОУРОВНЕВЫХ ЯЗЫКОВ ПРОГРАММИРОВАНИЯ ДЛЯ НОВОГО РАЗРАБАТЫВАЕМОГО ЯЗЫКА ПРОГРАММИРОВАНИЯ
}

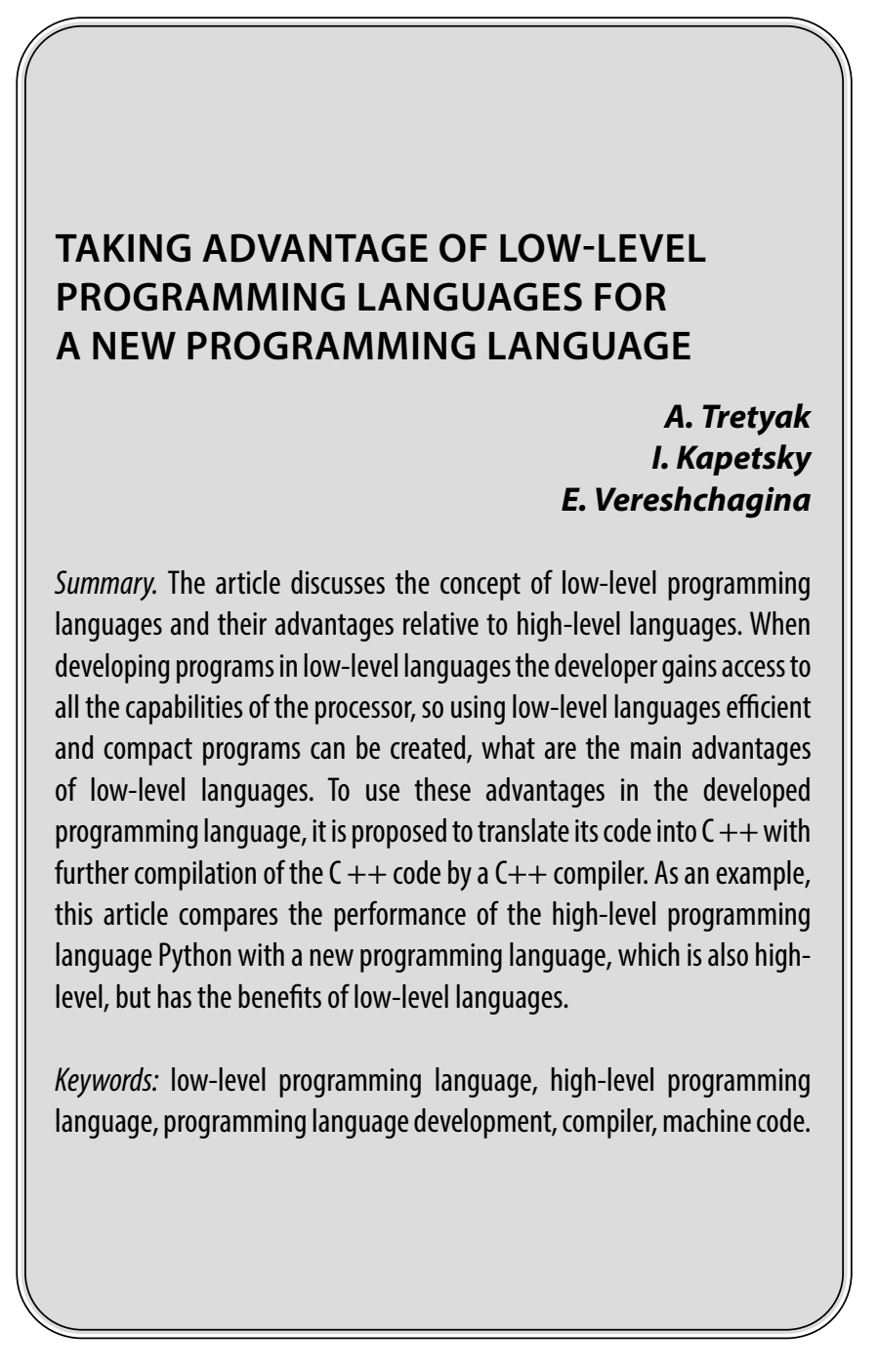

\section{Введение}

я зык программирования - это формальный язык, предназначенный для записи компьютерных программ [1]. Язык программирования определяет набор лексических, синтаксических и семантических правил, определяющих внешний вид программы и действия, которые выполнит компьютер под её управлением. Язык программирования предназначен для написания компьютерных программ, которые представляют собой набор правил, позволяющих компьютеру выполнить тот или иной вычислительный процесс, организовать управление различными объектами и т.п. [2, с. 7]. третьяк Александр Викторович

Аспирант, Дальневосточный федеральный университет, г. Владивосток alextretyak2@gmail.com

Капецкий Игорь Олегович

Старший преподаватель, Дальневосточный федеральный университет, г. Владивосток ikapeckij@mail.ru

Верещагина Елена Александровна

К.т.н., дочент, Дальневосточный федеральный университет, г. Владивосток everesh@mail.ru

Аннотация. В статье рассматривается понятие низкоуровневых языков программирования и их преимущества относительно высокоуровневых языков. Поскольку при разработке программ на языках низкого уровня разработчик получает доступ ко всем возможностям процессора, с помощью языков низкого уровня создаются эффективные и компактные программы, что является основными преимуществами низкоуровневых языков. Для использования данных преимуществ в разрабатываемом языке программирования предлагается производить его трансляцию В $C++$ с дальнейшей компиляцией кода $C++$ компилятором. В качестве примера приводится сравнение производительности высокоуровневого языка Pуthon с новым разрабатываемым языком программирования, который также является высокоуровневым, но обладает преимуществами низкоуровневых языков.

Ключевые слова: низкоуровневый язык программирования, высокоуровневый язык программирования, разработка языка программирования, компилятор, машинный код.

В настоящее время существуют тысячи языков программирования. Их можно классифицировать различными способами. Один из способов классификации по аппаратноориентированности и человекоориентированности - низкоуровневые и высокоуровневые языки программирования [3].

Низкоуровневый язык программирования (язык программирования низкого уровня) - язык для написания компьютерных программ, похожий на машинный код [4].

Высокоуровневый язык программирования (язык программирования высокого уровня) - язык для напи- 
сания компьютерных программ, который больше похож на человеческий, чем на компьютерный язык, за счет чего его легче понять программисту [5].

Целью данной работы является исследование преимуществ низкоуровневых языков программирования и использование этих преимуществ в новом разрабатываемом языке программирования.

\section{Преимушества низкоуровневых языков программирования}

С помощью языков низкого уровня создаются эффективные и компактные программы, поскольку разработчик получает доступ ко всем возможностям процессора. Под эффективной понимается программа, имеющая высокую скорость выполнения и малый объём занимаемой оперативной памяти, а под компактной - программа, имеющая небольшой размер скомпилированного исполняемого файла.

Среди недостатков низкоуровневых языков: необходимость высокой квалификации программиста ввиду сложности программ, труднопереносимость программ на устройство с другим типом процессора, значительное время разработки больших программ [6]. Всех этих недостатков лишены высокоуровневые языки программирования.

Таким образом, основными преимуществами низкоуровневых языков являются скорость выполнения и компактность программ.

\section{Низкоуровневые характеристики языка С}

Конструкции языка С близко сопоставляются типичным машинным инструкциям, благодаря чему он нашёл применение в проектах, для которых был свойственен язык ассемблера - в операционных системах, системном и прикладном программном обеспечении, а его синтаксис стал основой для таких языков программирования, как C++, C\#, Java и Objective-C [7].

C-подобные языки C и C++ наиболее близки к низкоуровневым языкам из всех прочих языков высокого уровня и имеют следующие характеристики.

Во-первых, язык С обладает портируемостью. Портируемость компилятора упрощает разработку компиляторов языка для новых платформ, а многообразие поддерживаемых компиляторами платформ избавляет разработчиков от необходимости переписывать прикладные программы для каждого компьютера.
Во-вторых, язык С позволяет работать с деталями реализации платформы: непосредственная работа с памятью и объектами в ней как с массивом байтов, возможность напрямую работать с адресами байтов и развитая арифметика указателей [8].

B-третьих, язык С достаточно близок к аппаратной части, поэтому он разделяет такие преимущества низкоуровневых языков, как скорость выполнения и компактность программ.

Вторая и третья представленные характеристики полностью соответствуют исключительно низкоуровневым языкам программирования.

\section{Пример ускорения \\ программного коАа}

Для использования преимуществ низкоуровневых языков программирования, таких как скорость выполнения и компактность программы, в разрабатываемом новом языке производится его трансляция в $\mathrm{C}++$ с дальнейшей компиляцией кода $\mathrm{C}++$ компилятором.

Однако во многом синтаксис языка Python удобнее для восприятия программистом, чем языков С и С++ (разработка на Python идёт быстрее, чем на многих других языках), а также чаще используется при решении таких задач как написание скриптов для обработки файлов и веб-запросов, для автоматизации задач в системном администрировании, а также для анализа больших данных. Также Python подходит и для создания прикладных приложений или игр [9]. Поэтому в разрабатываемом новом языке программирования синтаксис основывается на языке Python, но при этом сохраняются преимущества низкоуровневых языков, такие как скорость выполнения и малый объём занимаемой памяти.

Ниже представлен пример исходного кода утилиты, проверяющей парность скобок и кавычек во всех текстовых файлах в заданном каталоге.

Код на Python:

import sys, os

if _name__ ="'_main_':

for root, dirs, files in os.walk(sys.argv[1]):

for name in files:

if (name.endswith('.txt') and name not in ('last-message. txt', 'cur-message.txt', 'histedit-last-edit.txt')) or name. endswith(('.py,','hpp,','h,'.cpp')):

if $b^{\prime \prime} \backslash r^{\prime \prime}$ in open(os.path.join(root, name), 'rb').read(): "'"') 
filestr: str

try:

filestr $=$ open(os.path.join(root, name), ' $r$ ', encoding $=$ 'utf-8-sig').read()

except:

sys.exit("Exception while reading file '" + os.path. join(root, name) $\left.+{ }^{\prime \prime \prime \prime \prime}\right)$

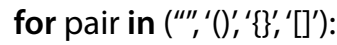

$\mathrm{i}=0$

while $\mathrm{i}<$ len(filestr):

if filestr[i] $==$ pair[0]:

start_i $=\mathbf{i}$

nesting_level $=1$

$\mathrm{i}+=1$

while True:

if $\mathrm{i}==$ len(filestr):

sys.exit("Balance check error in file "' + os.path.join(root, name) $\left.+{ }^{\prime \prime \prime \prime \prime}\right)$

$\mathrm{ch}=$ filestr[i]

$\mathrm{i}+=1$

if $\mathrm{ch}==$ pair[0]:

nesting_level $+=1$

elif $\mathrm{ch}==$ pair[1]:

if pair[0] $==^{\prime}('$ :

assert $\left.\left(\text { pair }[1]=={ }^{\prime}\right)^{\prime}\right)$

if filestr[i-1: i] ==':' and filestr $[i+1: i+3]=={ }^{\prime}(: ':$

assert $\left.\left(\text { filestr }[\mathrm{i}]==^{\prime}\right)^{\prime}\right)$

i += 2 \# пропускаем, чтобы смайлы:)(: не [ломали/] портили баланс

continue

nesting_level $-=1$

if nesting_level $==0$ :

break

elif filestr[i] $==$ pair[1]:

if pair[0] =='(':

assert $\left.\left(\text { pair }[1]==^{\prime}\right)^{\prime}\right)$

if filestr[i-1:i] == ':' and filestr[i+1: $i+3]=={ }^{\prime}\left(:^{\prime}:\right.$

assert(filestr[i] ==' $)^{\prime}$ )

i += 2 \# пропускаем, чтобы смайлы:)(: не [ломали/] портили баланс

continue

sys.exit("Balance check error in file "' + os.path.join(root, name) $\left.+{ }^{\prime \prime \prime \prime \prime}\right)$

else:

$\mathrm{i}+=1$

Код на новом языке программирования:

: start:

loop(_fname) fs: walk_dir(: argv[1], files_only'0B)

var root $=\mathrm{fs}$ : path: dir_name(_fname)

[String] dirs, files

if fs: is_dir(_fname) \{dirs [+]= fs: path: base_name( fname)\} else files [+]=fs: path: base_name(_fname)

loop(name) files if (name.ends_with('.txt') \& namelin ('last-message. txt', 'cur-message.txt', 'histedit-last-edit.txt')) | name.ends_ with(('.py,'.'hpp','.h',.'cpp'))

if "\} r \text { ".code in File(fs: path: join(root, name)).read_bytes() }

exit(' $\backslash r$ found in file "fs: path: join(root, name)"')

String filestr

exception.try

filestr = File(fs: path: join(root, name), 'r', encoding" utf-8-

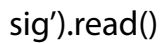

exception.catch

exit('Exception while reading file "fs: path: join(root, name) $\left.)^{\prime \prime \prime}\right)$

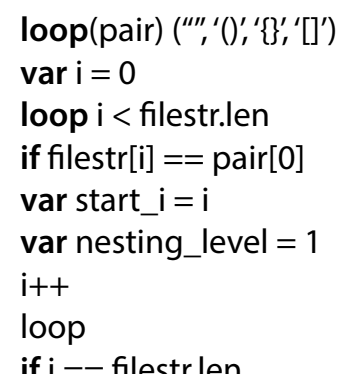

exit('Balance check error in file "fs: path: join(root, name) $\left.{ }^{\prime \prime \prime}\right)$

var $\mathrm{ch}=$ filestr $[\mathrm{i}$

i++

if $\mathrm{ch}==$ pair[0]

nesting_level++

else if $\mathrm{ch}==$ pair[1]

if pair[0] =='('

assert $\left.\left(\text { pair }[1]==^{\prime}\right)^{\prime}\right)$

if filestr $[i-1 .<\mathrm{i}]==^{\prime}::^{\prime} \&$ filestr $[\mathrm{i}+1 .<\mathrm{i}+3]==^{\prime}\left(:^{\prime}\right.$

assert(filestr[i] $\left.===^{\prime}\right)$ ')

$\mathrm{i}+=2$

loop.continue

nesting_level-

if nesting_level $==0$

loop.break

else if filestr[i] $==$ pair[1]

if pair $[0]=={ }^{\prime}('$

assert $\left.\left(\text { pair[1] }==^{\prime}\right)^{\prime}\right)$

if filestr[i $-1 .<\mathrm{i}]=={ }^{\prime}:{ }^{\prime} \&$ filestr $[i+1 .<\mathrm{i}+3]=={ }^{\prime}\left(\mathrm{\prime}^{\prime}\right.$

assert(filestr[i] $\left.\left.=={ }^{\prime}\right)^{\prime}\right)$

$\mathrm{i}+=2$

loop.continue

exit('Balance check error in file "fs: path: join(root, name) $)^{\prime \prime \prime}$ )

else

i++

Данная утилита запускалась в каталоге с исходным программным кодом транспайлера нового языка программирования [10]. Время работы данной утилиты на Python составило 4,5 секунды, а скомпилированного кода на новом языке - 0,26 секунд. Таким образом, ско- 
рость работы данной утилиты в 17 раз выше на новом языке программирования по сравнению с языком Python.

\section{Зак^ючение}

Основными преимуществами низкоуровневых языков программирования являются скорость выполнения и компактность программы; эти языки ориентированы на высокую производительность, эффективное использование аппаратных ресурсов компьютера, такие как процессор, память.
Для использования данных преимуществ в разрабатываемом языке программирования предлагается производить его трансляцию в $\mathrm{C}++$ с дальнейшей компиляцией кода $\mathrm{C}++$ компилятором.

Таким образом, можно сделать вывод, что используя трансляцию нового разрабатываемого языка программирования в C++, увеличивается производительность выполнения программного кода, а также используется малый объём занимаемой оперативной памяти.

\section{ЛИТЕРАТУРА}

1. Systems and software engineering — Vocabulary. URL: https://www.iso.org/standard/50518.html

2. Молдованова, О. В. Языки программирования и методы трансляции: учебное пособие / 0. В. Молдованова.— Новосибирск: Сибирский государственный университет телекоммуникаций и информатики, 2012.— 134 c.—URL: http://www.iprbookshop.ru/54809.html

3. Белова И. Язык программирования Python URL: https://youtu.be/617ybevPUKM?t=42

4. Low-level language URL: https://dictionary.cambridge.org/us/dictionary/english/low-level-language

5. High-level language URL: https://dictionary.cambridge.org/us/dictionary/ english/high-level-language

6. Языки программирования низкого уровня URL: https://studfile.net/preview/3972576/page:3/

7. Си (язык программирования) URL: https://ru.wikipedia.org/wiki/Си_(язык_программирования)

8. И всё же ( — низкоуровневый язык URL: https://habr.com/ru/company/badoo/blog/465429/

9. Где используется Python и в чём особенности этого языка URL: https://geekbrains.ru/posts/dlya-chego-nuzhen-yazyk-python

10. GitHub: 11l_to_cpp URL: https://github.com/111-lang/_11l_to_cpp

( ) Третьяк Александр Викторович ( alextretyak2@gmail.com ),

Капецкий Игорь Олегович ( ikapeckij@mail.ru ), Верещагина Елена Александровна ( everesh@mail.ru ).

Журнал «Современная наука: актуальные проблемы теории и практики»

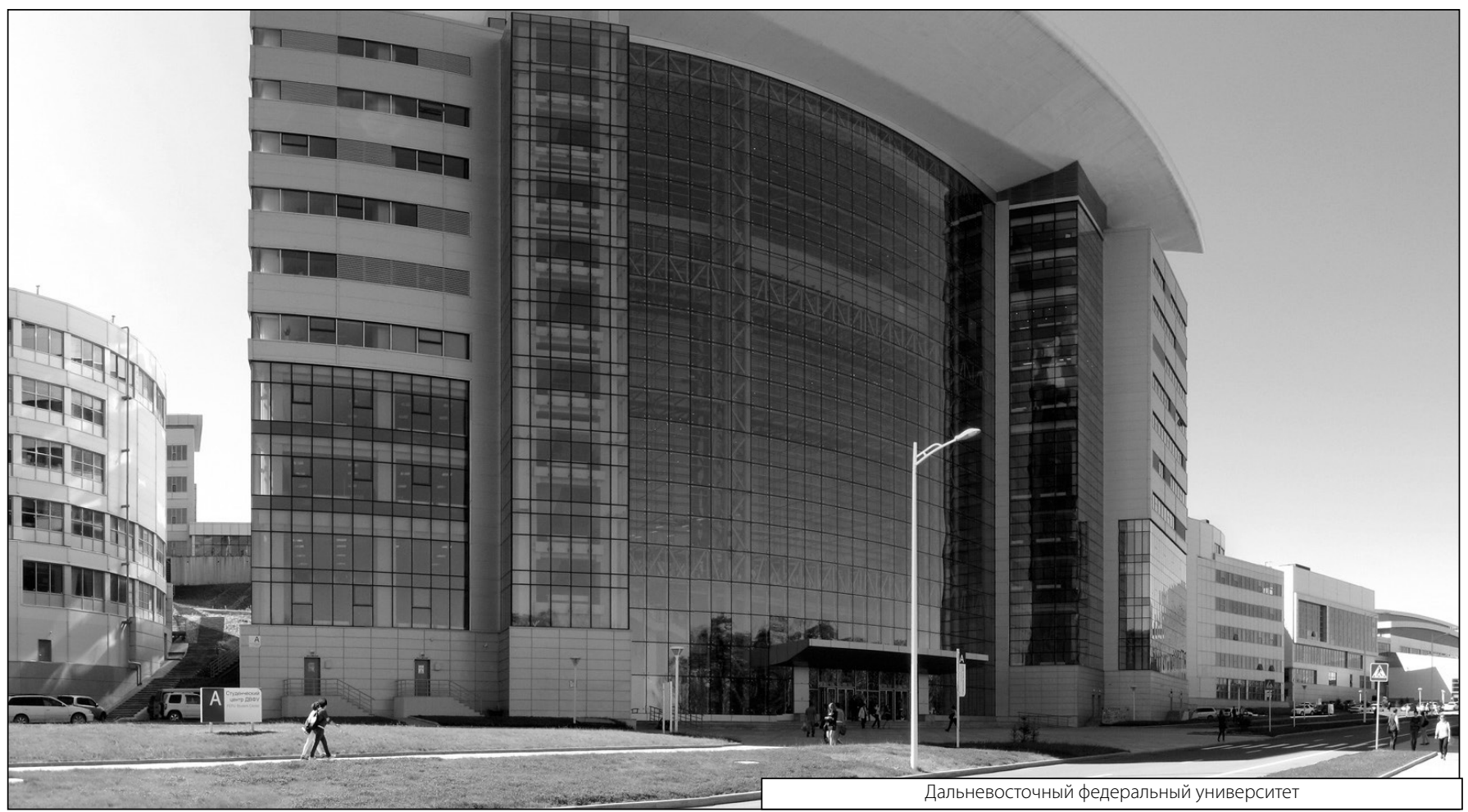

